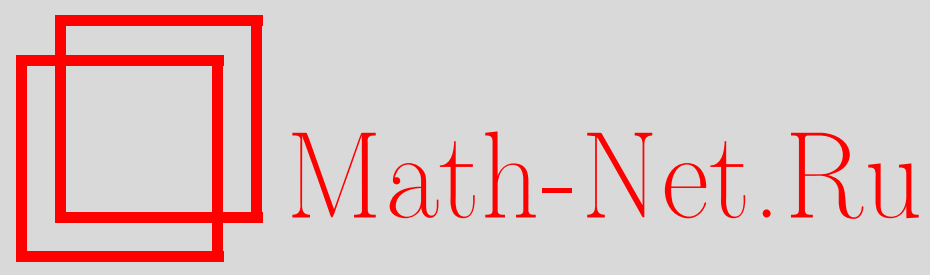

Б. С. Кашин, О нижних оценках $n$-членных приближений плоских выпуклых множеств и смежных вопросах, Матем. заметки, 2002, том 72, выпуск 4, 509-515

DOI: https://doi.org/10.4213/mzm440

Использование Общероссийского математического портала Math-Net.Ru подразумевает, что вы прочитали и согласны с пользовательским соглашением http://www.mathnet.ru/rus/agreement

Параметры загрузки:

IP : 54.157 .27 .8

26 апреля 2023 г., 09:19:34

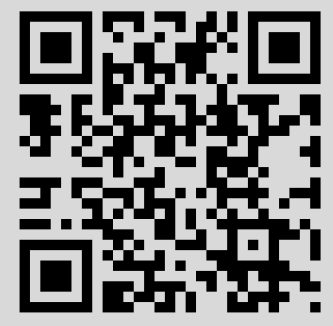




\section{О НИЖНИХ ОЦЕНКАХ $n$-ЧЛЕННЫХ ПРИБЛИЖЕНИЙ ПЛОСКИХ ВЫПУКЛЫХ МНОЖЕСТВ И СМЕЖНЫХ ВОПРОСАХ}

\section{Б. С. Кашин}

В работе установлены оценки снизу $n$-членных приближений в метрике $L^{2}\left(I^{2}\right)$ характеристических функций плоских выпуклых подмножеств квадрата $I^{2}$ по произвольньм ортогональным системам. Показано, что при $n \rightarrow \infty$ эти величины не могут убывать быстрее, чем $1 / n$.

Библиографоия: 12 названий.

Пусть $I^{d}=[0,1]^{d}$ - единичный куб в евклидовом пространстве $\mathbb{R}^{d}, d=1,2, \ldots$ Через $\mathfrak{M}_{d}$ обозначим совокупность всех вьпуклых подмножеств куба $I^{d}$ и положим

$$
K_{d}=\left\{\chi_{A}(x), A \in \mathfrak{M}_{d}\right\}
$$

где для $x \in I^{d}$

$$
\chi_{A}(x)=\left\{\begin{array}{l}
1, \text { если } x \in A, \\
0, \text { если } x \notin A .
\end{array}\right.
$$

В заметке установлены оценки снизу наилучших $n$-членных приближений семейства $K_{2}$ по произвольной ортонормированной системе в метрике гильбертова пространства $L^{2}\left(I^{2}\right)$. Кроме того, в связи с задачами теории приближения введена и для семейства $K_{2} \subset L^{2}\left(I^{2}\right)$ изучена одна количественная характеристика "массивности" произвольного подмножества элементов единичного шара гильбертова пространства.

1. Пусть $H$ - гильбертово пространство, $\Phi=\left\{\varphi_{j}\right\}$-ортонормированная система (о.н.с.) в $H, f \in H$.

ОПРЕДЕЛЕНИЕ 1. Наилучиим п-членным приближениемэлемента $f$ по системе $\Phi$ назьвается величина

$$
e_{n}(f, \Phi)=\inf _{P \in \Sigma_{n}}\|f-P\|_{H}
$$

где для $n=1,2, \ldots$

$$
\Sigma_{n} \equiv\left\{\sum_{j \in \Lambda} a_{j} \varphi_{j} ; \quad a_{j} \in \mathbb{R}, \quad \# \Lambda \leqslant n\right\},
$$

а через \# $\Lambda$ мы обозначаем число элементов в (конечном) множестве натуральных чисел $\Lambda$.

Работа выполнена при поддержке Российского фонда фундаментальных исследований, грант № 02-01-00315. 
Далее, если $\mathscr{F} \subset H$, то при $n=1,2, \ldots$

$$
e_{n}(\mathscr{F}, \Phi)=\sup _{f \in \mathscr{F}} e_{n}(f, \Phi)
$$

Аналогично определяются наилучшие $n$-членные приближения по произвольным системам элементов $\Phi$ в нормированных пространствах (см. подробнее [1], [2]). Активизация исследований $n$-членных приближений в последние годы в значительной степени связана с их приложениями в задачах сжатия изображений. При этом изучаются величины (2) для различных классов “достаточно хороших” функций, рассматриваемых как подмножества функционального нормированного пространства. Далеко не очевиден ответ на вопрос о том, какие функциональные классы $\mathscr{F}$ хорошо описьвают изображения, встречающиеся в реальной практике. С этой точки зрения представляется весьма естественной поставленная в 1993 г. С. В. Конягиным задача о возможном поведении при $n \rightarrow \infty$ величин

$$
e_{n}\left(K_{d}, \Phi\right), \quad d=1,2, \ldots, \Phi-\text { о.н.с. }
$$

При $d=1$ величины (3) могут убывать экспоненциально; это так, если $\Phi$ - система Хаapa. С другой стороны, в [3] установлено существование такой абсолютной постоянной $C>0$, что для любой о.н.с. $\Phi$

$$
e_{n}\left(K_{1}, \Phi\right) \geqslant C^{-n}, \quad n=1,2, \ldots
$$

Оказывается, что одномерньй случай является в определенном смысле вырожденньм и при $d>1$ характер поведения величин (3) меняется. В частности, имеет место

ТЕОРемА 1. Существует абсолютная постоянная $c>0$ такая, ито для любой о.н.с. $\Phi$ в $L^{2}\left(I^{2}\right)$ при $n=1,2, \ldots$ имеет место оценка

$$
e_{n}\left(K_{2}, \Phi\right) \geqslant \frac{c}{n}
$$

В доказательстве теоремы 1 нам потребуется

Теорема А [4]. Пусть для некоторого $m \in \mathbb{N}$ подмножсество $K$ гильбертова пространства $H$ содержит семейство $Q$ всех вериин некоторого $2 m$-мерного куба

$$
Q=\left\{\sum_{i=1}^{2 m} \varepsilon_{i}, \psi_{i}, \quad \varepsilon_{i}= \pm 1, \quad\left\{\psi_{i}\right\}_{i=1}^{2 m}-\text { o.н.c. }\right\} .
$$

Тогда для любой о.н.с. $\Phi$

$$
e_{m}(K, \Phi) \geqslant c_{1} m^{1 / 2}
$$

әде $c_{1}>0-$ абсолютная постоянная.

Положим

$$
\Delta_{2}\left(K_{2}\right)=\left\{f_{1}+f_{2}-2 f_{3}, f_{i} \in K_{2}, i=1,2,3\right\} .
$$

Легко видеть, что при $n=1,2, \ldots$

$$
e_{3 n}\left(\Delta_{2}\left(K_{2}\right), \Phi\right) \leqslant 4 e_{n}(K, \Phi) .
$$




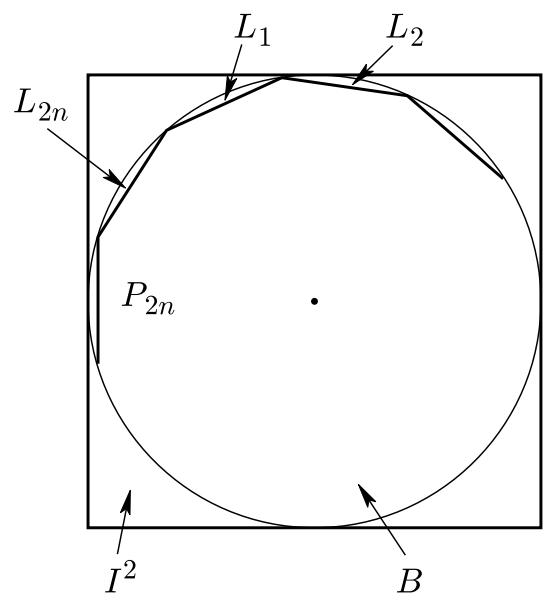

Рис. 1

Поэтому для доказательства теоремы 1 достаточно проверить, что при $n=2,3, \ldots$ для любой о.н.с. $\Phi$

$$
e_{n}\left(\Delta_{2}\left(K_{2}\right), \Phi\right) \geqslant c_{2} n^{-1}, \quad c_{2}>0
$$

Рассмотрим замкнутьй круг $B$ радиуса $1 / 2$ с центром в точке $(1 / 2,1 / 2) \in I^{2}$, и пусть $P_{2 n}$ - замкнутый правильный $2 n$-угольник, вписанньй в круг $B$ (см. рис. 1$)$.

Тогда разность $B \backslash P_{2 n}$ состоит из $2 n$ круговых сегментов $L_{1}, \ldots, L_{2 n}$. Для нас важно, что для любого набора натуральных чисел $\Lambda \subset\{1,2, \ldots, 2 n\}$ множество

$$
V(\Lambda)=P_{2 n} \cup\left\{\bigcup_{i \in \Lambda} L_{i}\right\}
$$

является вьпукльм множеством. Пусть $f_{1}=\chi_{B}, f_{2}=\chi_{P_{2 n}}, f_{3}=\chi_{V(\Lambda)}$, где $\Lambda \subset$ $\{1, \ldots, 2 n\}$. Тогда $f_{1}+f_{2}-2 f_{3} \in \Delta_{2}\left(K_{2}\right)$, причем

$$
f_{1}(x)+f_{2}(x)-2 f_{3}(x)= \begin{cases}+1, & \text { если } x \in L_{i}, i \notin \Lambda, \\ -1, & \text { если } x \in L_{i}, i \in \Lambda, \\ 0 & \text { иначе. }\end{cases}
$$

Таким образом, при любом выборе знаков $\left\{\varepsilon_{i}\right\}_{i=1}^{2 n}, \varepsilon_{i}= \pm 1$, имеет место включение

$$
\sum_{i=1}^{2 n} \varepsilon_{i} \chi_{L_{i}} \subset \Delta_{2}\left(K_{2}\right),
$$

причем функции $\chi_{L_{i}}, 1 \leqslant i \leqslant 2 n$, попарно ортогональны, имеют одинаковую норму в $L^{2}\left(I^{2}\right)$ и

$$
\left\|\chi_{L_{i}}\right\|_{L^{2}\left(I^{2}\right)} \geqslant c_{3} n^{-3 / 2} \text {. }
$$

Применение теоремы А с учетом соотношений (6), (7) приводит к оценке

$$
e_{n}\left(\Delta_{2}\left(K_{2}\right), \Phi\right) \geqslant c_{4} n^{-1}
$$


справедливой для любой о.н.с. Ф. Неравенство (4), а значит, и теорема 1 доказаны.

ЗАмечАниЕ. Оценка сверху вида $e_{n}\left(K_{2}, \Phi_{0}\right) \leqslant C n^{-1 / 2}$ в случае, когда $\Phi_{0}-$ кратная система Хаара, вытекает из результатов работы [5]. Отметим еще работу [6], в которой рассматриваются $n$-членные приближения характеристических функций плоских множеств с гладкой границей.

Пусть теперь $\Phi=\left\{\varphi_{j}\right\}-$ произвольный фрейм в гильбертовом пространстве $H$ и для $f \in H$

$$
\sigma_{n}(f, \Phi)=\inf _{\Lambda \subset \mathbb{N}, \# \Lambda \leqslant n}\left\|f-\sum_{j \in \Lambda}\left(f, \tilde{\varphi}_{j}\right) \varphi_{j}\right\|_{H},
$$

где $\left\{\tilde{\varphi}_{j}\right\}$ - фрейм, двойственньй к $\Phi$ (см. подробнее [7]). В [8] было проверено, что аналог теоремы А сохраняется для фреймов общего вида, если величину $e_{n}(K, \Phi)$ заменить на

$$
\sigma_{n}(K, \Phi)=\sup _{f \in K} \sigma_{n}(f, \Phi) .
$$

Поэтому рассуждения, приведенные в доказательстве теоремы 1 , показывают, что справедлива

ТЕОРема 2. Для произвольного фрейма $\Phi$ в $L^{2}\left(I^{2}\right)$ nри $n=1,2, \ldots$ имеет место неравенство

$$
\sigma_{n}\left(K_{2}, \Phi\right) \geqslant \frac{c}{n}, \quad c=c(\Phi)>0 \text {. }
$$

2. Пусть $K$ - подмножество единичного шара гильбертова пространства $H$. Для $n=1,2, \ldots$ положим

$$
O M_{n}(K)=n^{-1 / 2} \sup _{\substack{\left\{\psi_{j}\right\}_{j=1}^{n}-\text { o.н.с. } \\\left\{f_{j}\right\}_{j=1}^{n} \subset K}} \sum_{j=1}^{n}\left(\psi_{j}, f_{j}\right),
$$

где $(\cdot, \cdot)$ - скалярное произведение в $H$. Поведение последовательности

$$
\left\{O M_{n}(K)\right\}_{n=1}^{\infty},
$$

которую можно назвать “характеристикой ортомассивности" множества $K$, содержит полезную информацию о $K$. Легко видеть, что всегда

$$
\sup _{f \in K}\|f\|_{H} \leqslant O M_{n}(K) \leqslant n^{1 / 2}, \quad n=1,2, \ldots
$$

Нетрудно заметить также связь величин $O M_{n}(K)$ с $n$-поперечниками по Колмогорову множества $K$ в пространстве $H$.

Если $K=K_{1} \subset L^{2}(0,1)$, то

$$
O M_{n}\left(K_{1}\right) \asymp \log n, \quad n \rightarrow \infty .
$$

Этот результат есть следствие теоремы Меньшова-Радемахера (см. [9, с. 333, 336]). Более общо, если при $d=2,3, \ldots \pi_{d}$ есть множество характеристических функций $d$-мерных промежутков, лежащих в $I^{d}$, со сторонами, параллельными осям координат, то из результатов работ [10], [11] вытекает соотношение

$$
O M_{n}\left(\pi_{d}\right) \asymp(\log n)^{d}, \quad n \rightarrow \infty .
$$

Оказывается, что при $d>1$ последовательность $O M_{n}\left(K_{d}\right)$ растет существенно быстрее, чем в одномерном случае. 
TeOpema 3. $\Pi p u n=1,2, \ldots$

$$
O M_{n}\left(K_{2}\right) \geqslant c_{5} n^{1 / 6}, \quad c_{5}>0 .
$$

ДокАЗАТЕльСтво. Не ограничивая общности, считаем, что $n=m^{3}=2^{3 r}, r=$ $1,2, \ldots$ Внутри кольца

$$
\frac{1}{4} \leqslant\left|x-\left(\frac{1}{2}, \frac{1}{2}\right)\right| \leqslant \frac{1}{2}, \quad x \in I^{2}
$$

рассмотрим $p \geqslant c_{6} n^{2 / 3}, c_{6}>0$, колец $\Omega_{\nu}$ с центром в точке $(1 / 2,1 / 2) \in I^{2}$ таких, что гранища $\Omega_{\nu}$ состоит из окружностей $C_{\nu}^{\text {ext }}$ и $C_{\nu}^{\text {int }}$ радиусов $r_{\nu}^{\text {ext }}$ и $r_{\nu}^{\text {int }}$ соответственно, причем

1) $C_{\nu}^{\text {int }}=C_{\nu+1}^{\text {ext }}, \nu=1,2, \ldots, p-1$

2) если $\mathscr{D}_{\nu}$ - какой-то правильньй $m$-угольник, вписанный в $C_{\nu}^{\text {ext }}$, то

$$
\mathscr{D}_{\nu} \supset C_{\nu}^{\mathrm{int}}, \quad \nu=1, \ldots, p ;
$$

3) $0<c_{7} n^{-2 / 3} \leqslant r_{\nu}^{\text {ext }}-r_{\nu}^{\text {int }}<c_{8} n^{-2 / 3}, \nu=1, \ldots, p$.

Аналогично доказательству теоремы 1 , круговые секторы, на которые разбивается множество $\Omega_{\nu} \backslash \mathscr{D}_{\nu}$, обозначим $L_{i}^{\nu}, i=1, \ldots, m$.

Пусть $\left\{w_{j}\right\}_{j=1}^{m}-$ первые $m$ функций ортонормированной системы Уолша [9, с. 150]. Перенесем эти функции, принимаюшие значения +1 или -1 , на множество $\omega_{\nu}=\bigcup_{i=1}^{m} L_{i}^{\nu}$ (при $\nu=1,2, \ldots, p)$, положив

$$
\psi_{j}^{\nu}(x)= \begin{cases}\alpha_{\nu} w_{j}\left(\frac{i-1 / 2}{m}\right) & \text { для } x \in L_{i}^{\nu}, i=1, \ldots, m, \\ 0 & \text { для } x \notin \omega_{\nu},\end{cases}
$$

где $\alpha_{\nu}>0$ - нормируюший множитель такой, что $\left\|\psi_{j}^{\nu}\right\|_{L^{2}\left(I^{2}\right)}=1, \nu=1, \ldots, p, j=$ $1, \ldots, m$, причем нетрудно проверить, что

$$
\alpha_{\nu}=\left\{\operatorname{meas} \omega_{\nu}\right\}^{-1 / 2} \asymp n^{1 / 3}, \quad \nu=1, \ldots, p .
$$

Полученная в итоге система функций

$$
\left\{\psi_{j}^{\nu}, \quad \nu=1, \ldots, p, j=1, \ldots, m\right\}-\text { о.н.с. в } L^{2}\left(I^{2}\right) .
$$

Далее, пусть при $\nu=1, \ldots, p$ и $j=1, \ldots, m$

$$
Q_{j}^{\nu}=\bigcup_{i: \psi_{j}^{\nu}(x)>0 \text { для } x \in L_{i}^{\nu}} L_{i}^{\nu}, \quad E_{j}^{\nu}=\mathscr{D}_{\nu} \cup Q_{j}^{\nu} .
$$

Тогда (см. доказательство теоремы 1) $E_{j}^{\nu}$ - вьпуклое множество, т.е.

$$
f_{j}^{\nu}=\chi_{E_{j}^{\nu}} \in K_{2}, \quad \nu=1, \ldots, p, \quad j=1, \ldots, m .
$$


При этом (см. (10), (11))

$$
\sum_{(\nu, j)} \int_{I^{2}} f_{j}^{\nu} \psi_{j}^{\nu} d x=\frac{1}{2} \sum_{(\nu, j)} \int_{I^{2}}\left|\psi_{j}^{\nu}\right| d x=\frac{1}{2} \sum_{\nu=1}^{p} \sum_{j=1}^{m} \frac{1}{\alpha_{\nu}} \geqslant c_{9} n^{2 / 3}, \quad c_{9}>0
$$

т.е. $O M_{n}\left(K_{2}\right) \geqslant c_{9} n^{1 / 6}$, что и требовалось доказать.

Поясним, как могут использоваться оценки скорости роста последовательности (8). Пусть для данного $n$ набор элементов $\left\{f_{j}\right\}_{j=1}^{n} \subset K$ почти максимизирует выражение (7), т.е. найдется о.н.с. $\left\{\psi_{j}\right\}_{j=1}^{n}$, для которой

$$
\frac{1}{n^{1 / 2}} \sum_{j=1}^{n}\left(\psi_{j}, f_{j}\right) \geqslant \frac{1}{2} O M_{n}(K)
$$

Далее, пусть $\left\{\varphi_{i}\right\}_{i=1}^{s}-$ произвольная нормированная система элементов в $H$ такая, что при $j=1,2, \ldots, n$

$$
f_{j}=\sum_{i=1}^{s} c_{i}^{j} \varphi_{i}+\Delta_{j}, \quad \text { где }\left\|\Delta_{j}\right\|_{H} \leqslant n^{-1 / 2}, \quad j=1,2, \ldots, n .
$$

Тогда имеет место неравенство

$$
\sum_{i=1}^{s}\left(\frac{1}{n} \sum_{j=1}^{n}\left(c_{i}^{j}\right)^{2}\right)^{1 / 2} \geqslant \frac{1}{2} O M_{n}(K)-1
$$

Действительно,

$$
\frac{1}{2} O M_{n}(K) \leqslant \frac{1}{n^{1 / 2}} \sum_{j=1}^{n} \sum_{i=1}^{s} c_{i}^{j}\left(\psi_{j}, \varphi_{i}\right)+\frac{1}{n^{1 / 2}} \sum_{j=1}^{n}\left(\psi_{j}, \Delta_{j}\right),
$$

откуда, учитьвая (13) и неравенство Бесселя, мы находим

$$
\frac{1}{2} O M_{n}(K)-1 \leqslant \sum_{i=1}^{s}\left(\frac{1}{n^{1 / 2}} \sum_{j=1}^{n} c_{i}^{j} \psi_{j}, \varphi_{i}\right) \leqslant \sum_{i=1}^{s}\left(\frac{1}{n} \sum_{j=1}^{n}\left(c_{i}^{j}\right)^{2}\right)^{1 / 2}
$$

что и требовалось.

При наличии дополнительной информации о системе $\left\{\varphi_{i}\right\}_{i=1}^{s}$ можно в некоторых случаях выводить из (14) оценки снизу величин коэффициентов $c_{i}^{j}, 1 \leqslant i \leqslant s$, при некотором фиксированном $j$ (см. подробнее [3], [12], где неравенства, близкие к (14), применялись в частном случае, когда $K=K_{1} \subset L^{2}(0,1)$, а $\Phi$ - произвольная ортонормированная система в $\left.L^{2}(0,1)\right)$. 


\section{СПИСОК ЦИТИРОВАННОЙ ЛИТЕРАТУРЫ}

[1] DeVore R. A. Nonlinear approximation // Acta Numerica. 1998. V. 7. P. 51-150.

[2] Temlyakov V. N. Nonlinear Methods of Approximations. IMI Preprint 2001:09: University of South Carolina, 2001.

[3] Kashin B. S. On lower estimates for $n$-term approximation in Hilbert space // Approximation Theory. Volume Dedicated to B. Sendov. Sofia: Darba, 2002. P. 241-257.

[4] Кашин Б. С. Об аппроксимационных свойствах полных ортонормированных систем // Тр. МИАН. 1985. Т. 172. С. 187-191.

[5] Cohen A., DeVore R., Petrushev P., Xu H. Nonlinear approximation and the Space $B V\left(\mathbb{R}^{2}\right) / /$ Amer. J. Math. 1999. V. 121. P. 587-628.

[6] Donoho D. L. Sparse components of images and optimal atomic decompositions // Constr. Approx. 2001. V. 17. № 3. P. 353-382.

[7] Добеши И. Десять лекций по вейвлетам. М.-Ижевск: РХД, 2001.

[8] Кашин Б.С., Куликова Т. Ю. Замечание об аппроксимационных свойствах фреймов общего вида // Матем. заметки. 2002. Т. 72. № 2. С. 312-315.

[9] Кашин Б. С., Саакян А. А. Ортогональные ряды. 2-е изд. М.: АФЦ, 1999.

[10] Agnew R. P. On double orthogonal series // Proc. London Math. Soc. Ser. 2. 1932. V. 33. № 6 . P. 420-434.

[11] Moricz F. Multiparameter strong laws of large numbers. I // Acta Sci. Math. 1978. V. 40. P. $143-156$.

[12] Кашин Б. С. О коэффициентах разложения одного класса функций по полным системам // Сиб. матем. ж. 1977. Т. 18. №1. С. 122-131. 